\title{
Produtividade na pós-graduação stricto sensu em educação física do Rio de Janeiro: as publicações do corpo docente sob investigação
}

Productivity in post-graduation stricto sensu in physical education of Rio de Janeiro: publications of the teaching body under research

Productividad em la post-graduación stricto sensu em educaciónfísica del Río de Janeiro: las publicaciones del cuerpo docente bajo investigación

(iD) Débora Leonel Peluso

Universidade Federal do Rio de Janeiro, Rio de Janeiro, Rio de Janeiro, Brasil deboralpeluso@gmail.com

(iD) Alan Camargo Silva

Universidade do Estado do Rio de Janeiro, Rio de Janeiro, Rio de Janeiro, Brasil alan10@zipmail.com.br

(D) Felipe da Silva Triani

Universidade do Estado do Rio de Janeiro, Rio de Janeiro, Rio de Janeiro, Brasil felipetriani@gmail.com

iD Silvio de Cassio Costa Telles

Universidade do Estado do Rio de Janeiro, Rio de Janeiro, Rio de Janeiro, Brasil silviotelles@ufrj.br

iD Sílvia Maria Agatti Lüdorf Universidade Federal do Rio de Janeiro, Rio de Janeiro, Rio de Janeiro, Brasil silvialudorf@eefd.ufrj.br 
Resumo: Buscou-se investigar como ocorre a produção científica do corpo docente de diferentes subáreas, em especial a sociocultural e pedagógica, dos Programas de Pós- Graduação em Educação Física do Rio de Janeiro (PPGEF). Foram investigados dois PPGEF e a produção científica do respectivo corpo docente de 2013 a 2015. Detectou-se que a subárea biodinâmica prevalece em quantidade e qualidade de artigos, conforme critérios da CAPES. Entretanto, as vertentes sociocultural e pedagógica são representadas, principalmente, por meio de produções como livros e capítulos de livro.

Palavras-chave: Educação Física. Epistemologia. Pós-graduação. Docente.

Abstract: The objective of this study was to investigate how the scientific production of the teaching staff of different subareas, especially the sociocultural and pedagogical, of the Graduate Programs in Physical Education of Rio de Janeiro (GPPE). Two GPPE and the scientific production of the respective faculty from 2013 to 2015 were investigated. It was detected that the biodynamic subarea prevails in quantity and quality of articles, according to CAPES criteria. However, the sociocultural and pedagogical aspects are mainly represented through productions such as books and book chapters.

Keywords: Physical education. Epistemology. Postgraduate. Teacher.

Resumen: El objetivo de este estúdio fue investigar como ocorre la producción científica del cuerpo docente de diferentes subáreas, en especial, la sociocultural y pedagógica, de los Programas de Posgrado em Educación Física de Río de Janeiro (PPEF). Se investigó dos PPEFy la producción científica del respectivo cuerpo docente de 2013 a 2015. Detectó que la subárea biodinámica prevalece encantidad y calidad de artículos, conforme criterios de la CAPES. Sin embargo, las vertientes sociocultural y pedagógica son representadas, principalmente, por medio de producciones como libros y capítulos de libro.

Palabras clave: Educación física. Epistemología. Posgraduación. Enseñanza.

Submetido em: 10-03-2019

Aceito em: 17-03-2020 
Produtividade na pós-graduação stricto sensu em educação física do Rio de Janeiro... Débora Leonel Peluso - Alan Camargo Silva • Felipe da Silva Triani • Silvio de Cassio Costa Telles • Sílvia Maria Agatti Lüdorf

\title{
Introdução
}

Todo e qualquer campo, segundo Bourdieu (2004), se caracteriza por um espaço relativamente autônomo, que sobrevive conforme suas leis próprias. Referindo-se ao conceito bourdieusiano de campo científico, reconhece-se a Educação Física (EF) como um mundo social no qual estão inseridos os agentes e as instituições que produzem, reproduzem e difundem a ciência sob leis específicas. Trata-se de um campo de forças, mais precisamente de lutas, para conservar/transformar sua estrutura. É um jogo no qual suas próprias regras estão em jogo.

A moeda de alto valor nesse jogo do campo da EF, que pode ser denominada de capital científico, é atribuída pelo reconhecimento de competência e confere autoridade ao cientista, uma vez que "[...] os agentes caracterizados pelo volume de seu capital determinam a estrutura do campo [...]" (BOURDIEU, 2004, p. 24). Desse modo, na EF, regras definidas por determinados agentes influenciam eventuais escolhas ou mesmo atribuem níveis diferentes de importância a certos temas, além de produtos, tais como dissertações, teses, livros, artigos etc. Considerando-se as diferentes subáreas da EF, biodinâmica, sociocultural e pedagógica (MANOEL e CARVALHO, 2011), as formas de acumulação de capital científico eram peculiares, de acordo com Lüdorf e Castro (2017, p. 23):

\begin{abstract}
Enquanto as subáreas sociocultural e pedagógica eram, em grande medida, caracterizadas por teóricos de destaque, que lançavam livros de grande repercussão, muitas vezes reeditados e que se tornariam referências importantes para a Educação Física, os laboratórios de pesquisa na área da biodinâmica do movimento e seus líderes acumulavam capital científico, dentre outros modos, por meio dos artigos publicados, muitas das vezes, internacionalmente.
\end{abstract}

Nos últimos anos, contudo, há certo consenso de que o número de artigos publicados e de citações vem sendo considera- 
Produtividade na pós-graduação stricto sensu em educação física do Rio de Janeiro... Débora Leonel Peluso - Alan Camargo Silva • Felipe da Silva Triani • Silvio de Cassio Costa Telles • Sílvia Maria Agatti Lüdorf

do, também na EF, como indicador de capital científico (SILVA; SORIANO, 2014). Nesse sentido, é importante investigar como essas formas de acumulação de capital científico se processam em áreas caracterizadas pela pluralidade epistemológica, como a EF.

A EF pode ser considerada um campo científico recente. Os primeiros cursos de Mestrado e de Doutorado no Brasil foram respectivamente criados na década de 1970 e 1980 e, a partir de então, o campo passa por um processo de consolidação (TELLES; LÜDORF; GIUSEPPE, 2017). Na década de 1990, com o III Plano Nacional de Pós-Graduação, a pesquisa passou a ser voltada para ciência e tecnologia e ganhou relevância na Pós-Graduação (PG), enfatizando a formação de pesquisadores ao invés de professores para o ensino superior (DA SILVA; GONÇALVES-SILVA; MOREIRA, 2014), o que impactou substancialmente o campo da EF.

Os atuais critérios utilizados para a avaliação dos programas de PG são focados na produtividade, pautados por indicadores bibliométricos e pela internacionalização (TELLES; LÜDORF; GIUSEPPE, 2017). Vaz, Almeida e Bassani (2014) temem que a necessidade de publicar desenfreadamente possa resultar em um déficit na qualidade dos artigos, inibindo a criatividade e inovação das pesquisas. Lovisolo (2007) considera que os artigos são muitas vezes corretos, burocráticos e adequados aos parâmetros sugeridos pelo "sistema científico" atual, mas pouco originais. Ainda que haja críticas dos pesquisadores da EF acerca de um possível "produtivismo científico" na área, nota-se que os próprios agentes desse campo, por vezes, adequam-se ou assujeitam-se às lógicas de publicação. Por isso, Bourdieu (2008) aponta a necessidade do exercício de reflexividade no mundo científico a fim de que os agentes envolvidos tenham consciência das suas posições ou ações políticas diante da produção do conhecimento.

Esse cenário acadêmico do campo da EF, quanto à produtividade científica, implica diretamente nas subáreas dos PPGEF. Por um lado, há uma tendência crescente de credenciamento de orientadores participantes das linhas de pesquisa associadas às ciências biológicas e da saúde. Já nas linhas relacionadas às ciências 
Produtividade na pós-graduação stricto sensu em educação física do Rio de Janeiro...

Débora Leonel Peluso - Alan Camargo Silva • Felipe da Silva Triani • Silvio de Cassio Costa Telles • Sílvia Maria Agatti Lüdorf

humanas e sociais na EF, ocorre certo retraimento (RIGO, RIBEIRO; HALLAL, 2011).

As subáreas sociocultural e pedagógica (SCP) parecem sofrer enfraquecimento diante da política de avaliação da CAPES em relação à produtividade na PG na EF, o que tem dificultado o desenvolvimento de estudos nessa linha (Vilaça e Palma, 2013; Castro et al., 2017). Há certa concordância de que as produções oriundas dessas subáreas não devem ser avaliadas pelos mesmos parâmetros da biodinâmica (Fórum, 2015). Alguns pesquisadores ligados às subáreas SCP podem migrar para áreas onde suas produções são mais valorizadas, como na de educação (BAPTISTA; CASTRO; LÜDORF, 2017).

Diante desse quadro, questiona-se em que medida os Programas de Pós-Graduação de Educação Física (PPGEF) se comportam no que diz respeito à produção de estudos entre as subáreas, sobretudo as SCP. Mais especificamente, este artigo pretende centrar a análise no caso do Rio de Janeiro (RJ). Destaque-se que esse Estado já foi protagonista dos PPGEF, principalmente na década de 1990, quando os PPGEF ativos representavam a terça parte da produção total de dissertações e teses no Brasil. Ademais, colaborava substancialmente para as pesquisas nas subáreas SCP também, na década posterior (CASTRO et al., 2017; LÜDORF; CASTRO, 2017).

Abrir frentes de investigações locais para se conhecer o que está sendo produzido em termos teórico-metodológicos faz emergir uma problematização atual e preocupante quanto aos rumos da produtividade acadêmica em EF no Brasil. Assim, espera-se colaborar para a compreensão de determinadas relações entre produtividade e os PPGEF.

Desse modo, o objetivo do presente estudo é investigar como ocorreu a produção científica do corpo docente de diferentes subáreas, em especial as SCP, de PPGEF do RJ, no período compreendido de 2013 a 2015. 
Produtividade na pós-graduação stricto sensu em educação física do Rio de Janeiro... Débora Leonel Peluso - Alan Camargo Silva • Felipe da Silva Triani • Silvio de Cassio Costa Telles • Sílvia Maria Agatti Lüdorf

\section{Metodologia}

A fim de analisar as publicações científicas produzidas pelo corpo docente dos PPGEF do RJ, realizou-se, inicialmente, a consulta aos sites das instituições para se ter acesso ao quadro docente de cada programa do Estado. Os critérios para seleção dos PPGEF foram: a) ser reconhecido e recomendado pela CAPES; b) estar ativo no momento da pesquisa, no período de 2013 a 2015; c) possuir dissertações defendidas. O período em tela foi demarcado em virtude de, no momento da pesquisa, se configurar como triênio de avaliação para os PPG. Pretendeu-se, assim, ter um recorte preciso para possibilitar uma análise mais detida. No que se refere às dissertações defendidas, trata-se de um critério importante por revelar a vitalidade de um PPG (MOLINA NETO et al., 2006).

Assim, o presente estudo contou com dois PPGEF que, à época, estavam em funcionamento e que atendiam aos critérios: PPGEF da Universidade Federal do Rio de Janeiro (UFRJ) e Programa de Pós-Graduação em Ciências da Atividade Física da Universidade Salgado de Oliveira (UNIVERSO). Cabe ressaltar que o atual Programa de Pós-Graduação em Ciências do Exercício e do Esporte da Universidade do Estado do Rio de Janeiro não foi inserido no presente estudo porque teve início em 16 de abril de 2014, não atingindo os critérios de inclusão. Já o programa da Universidade Gama Filho foi extinto em 2014.

Após a seleção dos PPGEF e dos professores, a partir do seu nome completo e titulação, acessou-se a Plataforma Lattes, organizada pelo Conselho Nacional de Desenvolvimento Científico e Tecnológico ( $\left.\mathrm{CNPq}^{1}\right)$, em busca do Currículo Lattes de cada cientista. Feita a leitura de todos os currículos dos pesquisadores da UFRJ e da UNIVERSO, foi produzida uma listagem com as publicações de cada docente no triênio 2013-2015. Em relação aos artigos científicos, observaram-se os periódicos em que os professores publicaram e suas respectivas classificações.

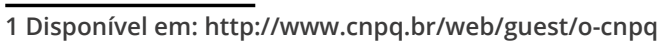


Produtividade na pós-graduação stricto sensu em educação física do Rio de Janeiro...

Débora Leonel Peluso - Alan Camargo Silva • Felipe da Silva Triani • Silvio de Cassio Costa Telles • Sílvia Maria Agatti Lüdorf

Os periódicos científicos eram, à época, avaliados de acordo com critérios de indexação e internacionalização, que compunham uma estratificação em 7 níveis: A1, A2, B1, B2, B3, B4, B5 e C. Esse sistema de avaliação de periódicos é denominado Qualis Periódico (Barata, 2016) e sofreu sensível alteração a partir dos novos critérios de avaliação da pós-graduação da CAPES e de nova estratificação, em 2019².

O levantamento dos estratos indicativos de qualidade ocorreu por meio do WebQualis ${ }^{3}$. A fim de observar possíveis mudanças no Qualis, buscou-se tomar ciência dos estratos dos periódicos de acordo com as listas de 2012 e 2014.

Considerando que cada estrato possui uma pontuação, o Documento de Área da EF (BRASIL, 2017) foi utilizado como base de consulta, e a pontuação relativa a cada nível de classificação dos periódicos é a que segue:

Quadro 1. Níveis de classificação dos periódicos e pontuação equivalente para a EF.

\begin{tabular}{|c|c|}
\hline Classificação & Pontos \\
\hline A1 & 100 \\
\hline A2 & 80 \\
\hline B1 & 60 \\
\hline B2 & 40 \\
\hline B3 & 20 \\
\hline B4 & 10 \\
\hline B5 & 5 \\
\hline C & 0 \\
\hline
\end{tabular}

Fonte: Brasil (2017).

Para a sistematização dos dados, foram elaboradas planilhas para cada PPGEF, que continham: nome completo de cada pesquisador; link de acesso ao Currículo Lattes; data da última atualização do currículo; referências completas das publicações no período de

2 A CAPES desenvolveu a proposta de um Qualis referência, na qual cada periódico teria uma única classificação, conforme a chamada área-mãe e outros critérios. Mais detalhes em: https://capes.gov.br/images/Relatorio_qualis_periodicos_referencia_2019/Relatorio_qualis_educacao_fisica.pdf

3 É o aplicativo que permite a classificação dos veículos de divulgação da produção intelectual dos programas de pós-graduação, bem como a divulgação do resultado dessa classificação (listas por área). 
Produtividade na pós-graduação stricto sensu em educação física do Rio de Janeiro...

Débora Leonel Peluso - Alan Camargo Silva • Felipe da Silva Triani • Silvio de Cassio Costa Telles • Sílvia Maria Agatti Lüdorf

2013-2015, em formato de artigo, livros e capítulos de livros; nível de estratificação e respectiva pontuação, atribuídos a cada um dos artigos.

Calculou-se, então, a soma de pontos de cada pesquisador da UFRJ e da UNIVERSO, por meio de suas publicações, e, por fim, realizou-se a soma das pontuações obtidas pelos pesquisadores das subáreas de cada programa.

\section{Resultados e Discussão}

A apresentação dos resultados e discussão foi realizada em dois momentos. Inicialmente, analisou-se a produção científica do corpo docente da UFRJ e da UNIVERSO quanto à quantidade de artigos e suas pontuações. Depois, o foco foi na produção científica dos pesquisadores das subáreas SCP, a fim de discutir a representatividade destes diante dos PPGEF.

\section{Produção científica do corpo docente da UFRJ e da UNIVERSO}

Em relação à produção científica, especialmente artigos publicados e análise das pontuações obtidas de cada docente, tem-se o seguinte panorama, conforme o Quadro 2: 
Produtividade na pós-graduação stricto sensu em educação física do Rio de Janeiro... Débora Leonel Peluso - Alan Camargo Silva • Felipe da Silva Triani • Silvio de Cassio Costa Telles • Sílvia Maria Agatti Lüdorf

Quadro 2. Pesquisadores do PPGEF da UFRJ e da UNIVERSO, quantidade de artigos publicados e suas pontuações.

\begin{tabular}{|c|c|c|c|c|c|}
\hline $\begin{array}{c}\text { Corpo Docente } \\
\text { da UFRJ }\end{array}$ & $\begin{array}{c}\text { Quantidade } \\
\text { de artigos } \\
\text { publicados } \\
\text { no triênio } \\
\mathbf{2 0 1 3 - 2 0 1 5}\end{array}$ & Qualis 2012 & $\begin{array}{c}\text { Quantidade de } \\
\text { Corpo Docente } \\
\text { da UNIVERSO }\end{array}$ & $\begin{array}{c}\text { Qrtigos publica- } \\
\text { dos no triênio } \\
\mathbf{2 0 1 3 - 2 0 1 5}\end{array}$ & Pontuação \\
Qualis 2012 \\
\hline Pesquisador 1 & 16 & 920 & Pesquisador 1 & 0 & 0 \\
\hline Pesquisador 2 & 14 & 690 & Pesquisador 2 & 10 & 305 \\
\hline Pesquisador 3 & 14 & 770 & Pesquisador 3 & 0 & 0 \\
\hline Pesquisador 4 & 31 & 2000 & Pesquisador 4 & 0 & 0 \\
\hline Pesquisador 5 & 3 & 160 & Pesquisador 5 & 8 & 185 \\
\hline Pesquisador 6 & 7 & 305 & Pesquisador 6 & 2 & 120 \\
\hline Pesquisador 7 & 8 & 400 & Pesquisador 7 & 3 & 130 \\
\hline Pesquisador 8 & 32 & 1745 & Pesquisador 8 & 10 & 550 \\
\hline Pesquisador 9 & 34 & 2160 & Pesquisador 9 & 42 & 2610 \\
\hline Pesquisador 10 & 11 & 720 & Pesquisador 10 & 73 & 3930 \\
\hline Pesquisador 11 & 11 & 325 & Pesquisador 11 & 16 & 765 \\
\hline Pesquisador 12 & 16 & 940 & TOTAL & $\mathbf{1 6 4}$ & $\mathbf{8 5 9 5}$ \\
\hline Pesquisador 13 & 5 & 280 & & & \\
\hline Pesquisador 14 & 9 & 360 & & & \\
\hline Pesquisador 15 & 43 & 2620 & & & \\
\hline Pesquisador 16 & 9 & 300 & & & \\
\hline Pesquisador 17 & 12 & 920 & & & \\
\hline Pesquisador 18 & 7 & 560 & & & \\
\hline TotaL & $\mathbf{2 8 2}$ & $\mathbf{1 6 1 2 0}$ & & & \\
\hline
\end{tabular}

Fonte: os autores (2020).

No PPGEF da UFRJ, a produção de artigos da biodinâmica é trinta vezes maior que a da SCP, isto é, no período compreendido entre 2013 e 2015, a biodinâmica publicou 282 artigos, enquanto a SCP, com um único representante, publicou 9 . Sem a necessidade de uma análise profunda, notável se torna a hegemonia da biodinâmica no PPGEF da UFRJ. Os pesquisadores ligados à subárea biodinâmica na UFRJ, além de publicarem mais, publicam em periódicos que pontuam mais. Cabe sinalizar que essa realidade coaduna com outras regiões brasileiras (QUADROS, AFONSO; RIBEIRO, 2013), como a região sul, em que há maior concentração de linhas de pesquisa e consolidação da biodinâmica em todos os PPGEF. 
Produtividade na pós-graduação stricto sensu em educação física do Rio de Janeiro...

Débora Leonel Peluso - Alan Camargo Silva • Felipe da Silva Triani • Silvio de Cassio Costa Telles • Sílvia Maria Agatti Lüdorf

Ao identificar a realidade dos 164 artigos do PPGEF da UNIVERSO, é interessante observar que o corpo docente é menor do que o da UFRJ, porém conta com mais pesquisadores das subáreas SCP comparados aos da biodinâmica. Contudo, observando-se as publicações científicas, constata-se que o quadro de predominância da subárea biodinâmica se repete. É importante lembrar que, anteriormente, foi publicado um estudo (MANOEL; CARVALHO, 2011) em que se detectava e problematizava a hegemonia da biodinâmica em praticamente todos os PPGEF do Brasil, e, passado alguns anos de discussão, o cenário parece ainda se manter.

Compreende-se, portanto, que a quantidade de pesquisadores de um PPGEF ligados a uma ou outra subárea não é fator determinante para definir o modo de funcionamento do programa e sim o poder atribuído aos pesquisadores detentores de capital científico. Essa espécie de capital é equivocadamente relacionada quase exclusivamente às publicações de "prestígio", como as que possuem maior reconhecimento de acordo com a pontuação recebida através do sistema de avaliação Qualis. Urge a necessidade de também lembrar o possível impacto dos índices bibliométricos que ajudam a legitimar e posicionar o pesquisador em dado lugar em um campo de saber (VIEIRA; WAINER, 2013).

Vilaça e Palma (2013) esclarecem que os docentes de PPGEF, além de publicarem certa quantidade de artigos, precisam atentar para o estrato do periódico, uma vez que a pontuação definirá se o artigo tem valor no mercado acadêmico. Para Telles, Lüdorf e Giuseppe (2017), publicar em periódicos com melhores estratos é condição indispensável para que os pesquisadores se mantenham credenciados nos programas.

Ao se comparar as instituições, destaca-se a proporcionalidade quanto ao número de pesquisadores e o número de artigos publicados. Enquanto na UFRJ, 18 pesquisadores publicaram 282 artigos, média de 15,6 manuscritos publicados por docente; na UNIVERSO, 11 pesquisadores publicaram 164 artigos, média de 14,90 . As pontuações dos artigos publicados seguiram a mesma tendência uma vez que, enquanto na UFRJ, houve um índice 
Produtividade na pós-graduação stricto sensu em educação física do Rio de Janeiro...

Débora Leonel Peluso - Alan Camargo Silva • Felipe da Silva Triani • Silvio de Cassio Costa Telles • Sílvia Maria Agatti Lüdorf

de aproximadamente 57,1 pontos para cada artigo publicado, na UNIVERSO, houve 52,4 pontos. Isso significa afirmar que, na realidade investigada, o número de professores não foi determinante para maior/melhor ou menor/pior produção de artigos científicos.

Foi possível identificar uma característica importante e preocupante quanto ao nível de classificação produzido entre as subáreas na UNIVERSO. Embora os pesquisadores da biodinâmica estejam em desvantagem numérica em comparação aos das SCP, publicaram uma quantidade de artigos seis vezes maior do que a quantidade dos artigos dos demais pesquisadores. A pontuação referente às publicações dos pesquisadores da biodinâmica atinge 7855, enquanto os artigos produzidos pelos pesquisadores das SCP somam uma pontuação de 740, quase dez vezes menor. Essa dinâmica reforça os argumentos de Triani e Telles (2017) de que o número de docentes nas subáreas SCP é inversamente proporcional à pontuação do programa e, consequentemente, sua nota.

Analisando-se os periódicos científicos em que os pesquisadores das subáreas SCP publicam, foram encontradas revistas que possuem estrato baixo, visto que não atendem a alguns critérios priorizados pelo Qualis Periódico, como a internacionalização. Mas é entendido que as pesquisas de cunho SCP tenham pouca inserção em periódicos internacionais, porque, muitas das vezes, referem-se a realidades locais, o que não desperta tanto interesse em pesquisadores de outros países, que buscam resultados mais abrangentes e padronizados, como alguns estudos biodinâmicos apresentam. Além disso, o potencial de internacionalização dos estudos da biodinâmica é maior do que os das SCP (TRIANI; TELLES, 2017).

O baixo potencial de generalização implica na necessidade de pesquisadores brasileiros do campo da EF investirem em periódicos nacionais. No entanto, Lazzarotti Filho (2018) assinala que, dos 12 principais periódicos brasileiros, somente 9 estão mais associados às subáreas SCP, sendo que dois deles aceitam somente manuscritos em inglês. Além disso, a Revista Brasileira de Ciências do Esporte, que antes aceitava trabalhos em três idiomas, agora, para uma das subáreas, aceitará somente em inglês, desvelando 
Produtividade na pós-graduação stricto sensu em educação física do Rio de Janeiro...

Débora Leonel Peluso - Alan Camargo Silva • Felipe da Silva Triani • Silvio de Cassio Costa Telles • Sílvia Maria Agatti Lüdorf

que há uma tendência nesse sentido, considerando os critérios de avaliação de periódicos científicos (MASCARENHAS; LAZZAROTTI FILHO; VIANNA, 2018). Esse fato implica no grande número de trabalhos sendo produzidos nos PPGEF para um baixo número de periódicos para veiculação dessas produções.

O fato de não ter sido publicado em um periódico internacional não significa que o estudo não tenha sido elaborado com rigor, seriedade, ética e que, principalmente, não tenha importância para a comunidade. Contudo, como salientam Andrade e Galembeck (2009), não é o impacto social e a qualidade de produção do conhecimento científico que distinguem os periódicos e sim a visibilidade deles. Desse modo, as publicações científicas se transformam em uma espécie de moeda, símbolo de poder e determinante para a posição social do pesquisador. Além disso, segundo o critério de avaliação adotado pela CAPES (BRASIL, 2016), internacionalizar a produção significa aumentar a visibilidade por meio dos indicadores bibliométricos, sendo assim, essa seria a produção mais "qualificada".

Outro ponto que chama a atenção é que os professores bolsistas por produtividade de ambos os programas são vinculados à subárea biodinâmica. Além do capital científico, acumulam um capital econômico, consequentemente de suas produções. Constatação esta que se alinha ao pensamento de Bourdieu (2004, p. 25) quando afirma que "[...] as oportunidades que um agente singular tem de submeter as forças sobre o campo aos seus desejos são proporcionais à sua força sobre o campo, isto é, ao seu capital de crédito científico [...]".

Nota-se, portanto, que, embora o trabalho comparativo do presente estudo seja microscópico e local, as preocupações científicas, em geral, estão diretamente relacionadas à política de produção apresentada na última avaliação (BRASIL, 2017). Essa realidade, de abrangência nacional, possivelmente influencia na escolha do tema a ser pesquisado e o tipo de pesquisa a ser desenvolvido. Corre-se o risco, embora possa ser um argumento controverso, de que os empreendimentos de pesquisa sejam influenciados mais pela probabilidade de ser publicado do que propriamente pela ne- 
Produtividade na pós-graduação stricto sensu em educação física do Rio de Janeiro...

Débora Leonel Peluso - Alan Camargo Silva • Felipe da Silva Triani • Silvio de Cassio Costa Telles • Sílvia Maria Agatti Lüdorf

cessidade de avanços no conhecimento de um assunto específico (MATTOS, 2017). Kokubun (2003, p. 11) já alertava que a formação de recursos humanos para o magistério superior vem mascarando a função "talvez mais importante da PG que é a de capacitar recursos humanos qualificados para a produção de conhecimentos relevantes e inovadores para o desenvolvimento da área". Adicionalmente, Pires e Poffo (2018) ressaltam que essa prática é endossada pelo Sistema Nacional de Pós-Graduação na medida em que valoriza o célebre "publicar ou perecer".

Na perspectiva de Bourdieu (2004), o capital científico se caracteriza por um tipo específico de capital que atribui prestígio ao cientista. Quanto maior o capital científico de um pesquisador, maior será o seu reconhecimento e prestígio na academia. Justifica-se, assim, dentre outros fatores, a corrida no mundo acadêmico atual pela produtividade. A publicação de artigos, em especial, se constitui como um forte indicador de capital científico, representando poder específico num dado campo, agregando valor em torno de um autor, grupo de pesquisa, laboratório ou Universidade, como forma de reconhecimento do trabalho realizado e da validação desse conhecimento.

\section{Produção científica do corpo docente das subáreas SCP da UFRJ e da UNIVERSO}

Uma análise mais voltada às produções dos docentes ligados às subáreas SCP permite visualizar os seguintes dados, sistematizados na Tabela 1: 
Produtividade na pós-graduação stricto sensu em educação física do Rio de Janeiro... Débora Leonel Peluso - Alan Camargo Silva • Felipe da Silva Triani • Silvio de Cassio Costa Telles • Sílvia Maria Agatti Lüdorf

Tabela1. Pesquisadores das subáreas SCP dos PPGEF da UFRJ e UNIVERSO, quantidade de artigos publicados e suas pontuações pelo Qualis 2012 e Qualis2014.

\begin{tabular}{cccc}
\hline Corpo Docente & $\begin{array}{c}\text { Quantidade de artigos } \\
\text { publicados no triênio } \\
\text { 2013-2015 }\end{array}$ & $\begin{array}{c}\text { Pontuação } \\
\text { Qualis 2012 }\end{array}$ & $\begin{array}{c}\text { Pontuação } \\
\text { Qualis 2014 }\end{array}$ \\
\hline UNIVERSO & 0 & 0 & 0 \\
\hline Pesquisador 1 & 10 & 305 & 280 \\
Pesquisador 2 & 0 & 0 & 0 \\
Pesquisador 3 & 0 & 0 & 0 \\
Pesquisador 4 & 8 & 185 & 125 \\
Pesquisador 5 & 2 & 120 & 120 \\
Pesquisador 6 & 3 & 130 & 125 \\
Pesquisador 7 & \multicolumn{3}{c}{} \\
\hline UFRJ & 9 & $\mathbf{1 0 4 0}$ & $\mathbf{8 8 5}$ \\
\hline Pesquisador 8 & $\mathbf{3 2}$ & \\
\hline TOTAL & \multicolumn{2}{c}{ Fonte: os autores (2020). }
\end{tabular}

Os pesquisadores das subáreas SCP sofrem desvantagens por serem avaliados pelos mesmos critérios da biodinâmica, na medida em que a internacionalização é um fator determinante (BRASIL, 2017), característica que não é própria das subáreas SCP (CASTRO et al, 2017; TRIANI; TELLES, 2017). Além disso, há indícios de que essa situação se agrave cada vez mais, pois, em 2014, o Qualis Periódico atualizou a tabela de estratificação dos periódicos e, para as subáreas SCP, as modificações não foram proveitosas.

A soma da pontuação equivalente à produção de artigos dos pesquisadores das subáreas SCP das duas instituições, UFRJ e UNIVERSO, quando calculada pelo Qualis Periódico de 2014, cai em relação ao Qualis 2012. Com as alterações de estrato de alguns dos periódicos, fica ainda mais trabalhoso chegar a uma pontuação que colabore na avaliação do programa e, consequentemente, na sobrevivência das subáreas SCP nos programas (LÜDORF; CASTRO, 2017). Observa-se, por exemplo, a estratificação da Revista Motriz ${ }^{4}$, periódico na qual há inserção de estudos socio-

$\overline{4 \text { Mais informações: }}$ http://www.scielo.br/scielo.php?script=sci_serial\&pid=1980-6574\&lng=pt\&nrm=iso 
Produtividade na pós-graduação stricto sensu em educação física do Rio de Janeiro...

Débora Leonel Peluso - Alan Camargo Silva • Felipe da Silva Triani • Silvio de Cassio Costa Telles • Sílvia Maria Agatti Lüdorf

culturais e pedagógicos, que era A2, de acordo com o Qualis 2012, equivalente a 80 pontos, e passou a B1, o que corresponde a 60 pontos.

Essa preocupação quanto ao Qualis Periódico não é exclusiva do estudo em tela, tendo em vista que tem sido apresentada por diversos autores e vem sendo enfatizada com o passar dos anos, ganhando foco em eventos acadêmicos e periódicos científicos da área. Pesquisadores das subáreas SCP da EF argumentam que a continuidade dessas vertentes de estudo da EF está ameaçada diante da redução significativa de dissertações e teses, diagnosticada por meio da comparação das avaliações de área dos triênios 2001-2003 e 2010-2012 (FÓRUM, 2015). Vaz, Almeida e Bassani (2014) afirmam que pode ocorrer a extinção gradual dos docentes da área SCP do âmbito dos PPGEF.

Da Silva, Gonçalves-Silva e Moreira (2014) ressaltam que a produção científica se configura como material de grande relevância, pois brinda a sociedade com o "acesso aos avanços, retrocessos e limitações do conhecimento, permitindo que esses sejam questionados, refletidos, reavaliados, como também aplicados em intervenções cotidianas [...] (p. 1426)". Porém, a incidência política e exigências no mundo científico parecem iniciar uma distorção de valores no âmbito acadêmico, que mereceria análise mais detida no campo da EF, mas de certa maneira já alertada por Kunz (2012, p. 9): “[...] a questão crucial sobre o que se está pesquisando/escrevendo é substituída pela pergunta: qual o Qualis da revista em que se publica?".

Outro aspecto a ser destacado é que alguns professores não publicaram artigos no período analisado, mas, em contrapartida, produziram livros e capítulos de livro, como poderá ser visualizado na Tabela 2. Ainda que livros e capítulos de livro sejam dos principais meios de veiculação da produção científica em Ciências Humanas (TRIANI; TELLES, 2017), é necessário um olhar mais atento a esses dados. Especificamente em relação aos pesquisadores 1, 3 e 4, que não publicaram artigos, algumas interpretações podem ser esboçadas a partir da análise dos respectivos Currículos 
Produtividade na pós-graduação stricto sensu em educação física do Rio de Janeiro... Débora Leonel Peluso - Alan Camargo Silva • Felipe da Silva Triani • Silvio de Cassio Costa Telles • Sílvia Maria Agatti Lüdorf

Lattes. Dois desses professores vivenciaram praticamente todo o período de existência da pós-graduação em EF no Brasil, nesse, o PPGEF da Universo, ou em outros programas, o que sugere que podem ter construído capital científico à revelia das recentes políticas de produtividade ou, ainda, talvez não se interessem por "jogar o jogo". Já o outro docente dedicou-se, no triênio investigado, a cargos administrativos, talvez acarretando em certo distanciamento da pesquisa, principalmente ao se considerar o contexto da universidade privada, como é o caso.

Tabela 2. Pesquisadores das subáreas SCP dos PPGEF da UFRJ e da UNIVERSO e quantidade de suas publicações em formato de livros e capítulos de livro no triênio 2013-2015.

Pesquisadores das subáreas

Sociocultural e Pedagógica
Livros publicados no triênio 2013-2015
Capítulos de livro publicados no triênio

2013-2015

\section{UNIVERSO}

\begin{tabular}{lccc}
\hline & Pesquisador 1 & 1 & 3 \\
& Pesquisador 2 & 3 & 13 \\
& Pesquisador 3 & 0 & 0 \\
& Pesquisador 4 & 0 & 1 \\
& Pesquisador 5 & 4 & 7 \\
& Pesquisador 6 & 1 & 7 \\
& Pesquisador 7 & 2 & 3 \\
\hline UFRJ & & 0 & 1 \\
\hline \multicolumn{2}{c}{ Pesquisador 8 } & 11 & 35 \\
\hline
\end{tabular}

Fonte: os autores (2020).

Os pesquisadores da EF, que representam as subáreas SCP, podem ser considerados, pelos termos de Bourdieu (2004), como possíveis agentes de transformação, embora não se possa descartar a interpretação de que esses mesmos pesquisadores reiterem a lógica "produtivista" de outro modo. De acordo com o autor, os agentes sociais não são partículas passivamente conduzidas pelas forças do campo. Eles têm maneiras de ser, que podem levá-los a resistir, a opor-se, a lutar e tentar transformar, ajustar as estru- 
Produtividade na pós-graduação stricto sensu em educação física do Rio de Janeiro...

Débora Leonel Peluso - Alan Camargo Silva • Felipe da Silva Triani • Silvio de Cassio Costa Telles • Sílvia Maria Agatti Lüdorf

turas em razão de suas disposições. Continuar produzindo livros e capítulos de livro mesmo sabendo que esse tipo de publicação pode ser menos valorizado em relação ao artigo na avaliação da PG, é uma maneira de demonstrar a característica da subárea de produzir e divulgar o conhecimento.

Aqui, não se pretende deslindar as razões específicas da baixa representatividade na produção científica das subáreas SCP que, por vezes, ocorre no PPGEF, pois tal realidade pode estar associada a uma série de fatores, como: políticas da CAPES para a PG, tempo necessário do empreendimento de pesquisa qualitativa, questões políticas internas, comprometimento com outras dimensões da Universidade, como administrativa ou de extensão etc. Muitas vezes, o foco de produção dos docentes ligados às subáreas SCP não está voltado especificamente à pesquisa, ou ainda, à produção de artigos científicos, mas a outras demandas de ordem profissional ou pessoal. Isso pode ser visto quando, antigamente, privilegiava-se mais a docência do que o âmbito da pesquisa no sentido de produção de artigos científicos (RESENDE; VOTRE, 2003).

De toda forma, os dados aqui apresentados apontam para uma predominância da biodinâmica no campo da produção de conhecimento científico nos PPGEF do RJ e, consequentemente, os agentes bem posicionados dentro do campo são os pesquisadores ligados a essa subárea, corroborando o estudo de Castro et al. (2017). Segundo Bourdieu (2004, p. 29), "[...] quanto mais as pessoas ocupam uma posição favorecida na estrutura, mais elas tendem a conservar ao mesmo tempo a estrutura e sua posição".

Como estratégia para a conservação de suas posições, os pesquisadores se vêm na obrigação de manter um ritmo intenso de produção de artigos, o que pode gerar o movimento da produtividade para o produtivismo. Dessa maneira, a PG não estaria se tornando uma empresa gestora de artigos? Sendo como uma empresa que não se arrisca para minimizar as chances de fracasso, será que o modo atual de produção de conhecimento em EF na PG não estaria inibindo a criatividade e a inovação? 
Produtividade na pós-graduação stricto sensu em educação física do Rio de Janeiro...

Débora Leonel Peluso - Alan Camargo Silva • Felipe da Silva Triani • Silvio de Cassio Costa Telles • Sílvia Maria Agatti Lüdorf

Caberia retomar e refletir sobre o argumento de Bourdieu (2004), de que deve haver um equilíbrio entre a ciência pura, totalmente livre de qualquer necessidade social, e a ciência escrava, sujeita a todas as demandas político-econômicas.

\section{Conclusão}

Ao analisar a produção científica dos pesquisadores que constituem os corpos docentes dos PPGEF da UFRJ e da UNIVERSO, verificou-se que, em ambas instituições, os pesquisadores da biodinâmica produziram mais artigos e atingiram uma pontuação muito maior do que a dos pesquisadores das subáreas SCP.

Nos PPGEF do RJ, existe espaço para o desenvolvimento de estudos socioculturais e pedagógicos, embora haja um ambiente aparentemente favorável à construção de estudos biodinâmicos, considerando as políticas de avaliação. Caracterizados como agentes de conservação, a princípio, os pesquisadores dessa subárea seriam detentores de capital científico, o que os colocaria em uma posição privilegiada na estrutura do campo da EF. Como estratégia de conservação dessa estrutura e de suas posições, há necessidade de manter um ritmo acelerado de produção de artigos em estratos elevados.

Em relação aos pesquisadores das subáreas SCP, em sua luta para se manterem no campo ou eventualmente transformá-lo, dificilmente se adequam à estrutura e tentam demonstrar suas especificidades, como a produção científica veiculada através de livros e capítulos de livro, um dos modos privilegiados de divulgação de pesquisas em Ciências Humanas e Sociais. Entretanto, ressalta-se que, por vezes, a produção de livros e capítulos pode ser financiada pelos próprios pesquisadores, o que pode sugerir também um movimento substitutivo do "publicar ou perecer" por "pagar para publicar".

Ainda assim, observa-se que, nesse contexto, o RJ perde o protagonismo no que se refere às vertentes SCP, simbolizando a difi- 
Produtividade na pós-graduação stricto sensu em educação física do Rio de Janeiro...

Débora Leonel Peluso - Alan Camargo Silva • Felipe da Silva Triani • Silvio de Cassio Costa Telles • Sílvia Maria Agatti Lüdorf

culdade de sustentação que tais subáreas enfrentam na atualidade no campo científico da EF e o consequente enfraquecimento de estudos nessas vertentes.

Essa é apenas uma das variadas reflexões que estudos locais como este podem suscitar sobre a presente temática. Urge conhecer o panorama atual dos PPGEF em outras regiões do Brasil a fim de compreender os caminhos que a área vem trilhando no âmbito acadêmico e científico.

\section{Referências}

ANDRADE, J. B.; GALEMBECK, F. Qualis: quo vadis? Editorial. Química Nova, São Paulo, v. 32, n. 1, p. 1, 2009.

BARATA, R. C. B. Dez coisas que você precisa saber sobre o Qualis. Revista Brasileira de Pós-Graduação, Brasília, v. 13, n. 30, p. 13-40, 2016.

BAPTISTA, G. G.; CASTRO, P. H. Z.; LÜDORF, S. M. A. “Educação do corpo" e campo científico: da fluidez do conhecimento às lutas simbólicas. Revista Brasileira de Ciências do Esporte, Campinas, v. 3, n. 4, p. 330-37, 2017.

BRASIL. Ministério da Educação. Relatório de Avaliação Educação Física: avaliação quadrienal. Brasília: DF, 2017.

BOURDIEU, P. Os usos sociais da ciência: por uma sociologia clínica do campo científico. São Paulo: Cortez, 2004.

BOURDIEU, P. Por uma sociologia da ciência. Lisboa: Edições 70, 2008.

CASTRO, P. H. Z. et al. A produção científica em Educação Física de 2001 a 2010: caminhos da construção de um campo. Movimento, Porto Alegre, v. 23, m. 3, p. 869-888, 2017.

DA SILVA, J. V. P.; GONÇALVES-SILVA, L. L.; MOREIRA, W. W. Produtivismo na Pós-Graduação. Nada é tão ruim, que não possa piorar. É chegada a vez dos orientandos! Movimento, Porto Alegre, v. 20, n. 4, p. 1423-1445, 2014. 
Produtividade na pós-graduação stricto sensu em educação física do Rio de Janeiro... Débora Leonel Peluso - Alan Camargo Silva • Felipe da Silva Triani • Silvio de Cassio Costa Telles • Sílvia Maria Agatti Lüdorf

\section{FÓRUM DE PESQUISADORES DAS SUBÁREAS SOCIOCULTURAL} E PEDAGÓGICA. Cenários de um descompasso da Pós-Graduação em Educação Física e demandas encaminhas à CAPES. 2015. Disponível em: http://www.cbce.org.br/noticias-detalhe. php?id=1074. Acesso em: 1/4/2015.

KOKUBUN, E. Pós-Graduação em educação física no Brasil: indicadores objetivos dos desafios e das perspectivas. Revista Brasileira de Ciências do Esporte, Campinas, v. 24, n. 2, p. 9-26, 2003.

KUNZ, E. Pós-Graduação em Educação Física no Brasil: o fenômeno da hiperprodutividade e formação cultural. Kinesis, Santa Maria, v. 30, n. 1, p. 1-13, 2012.

LAZZAROTTI FILHO, A. O periodismo científico da educação física brasileira. Motrivivência, Florianópolis, v. 20, n. 54, p. 35-50, 2018.

LOVISOLO, H. A política de pesquisa e a mediocridade possível. Revista Brasileira de Ciências do Esporte, São Paulo, v. 24, n. 2, p. 97-114, 2003.

LOVISOLO, H. Levantando o sarrafo ou dando tiro no pé: critérios de avaliação e qualis das Pós-graduações em Educação Física. Revista Brasileira de Educação Física e Esporte, São Paulo, v. 29, n. 3, p. 23-34, 2007.

LÜDORF, S. M. A.; CASTRO, P. H. Realidades da pós-graduação em educação física: manutenção ou desmonte das subáreas sociocultural e pedagógica? In: TELLES, S.; LÜDORF, S.; GIUSEPPE, E. Pesquisa em educação física: perspectivas sociocultural e pedagógica em foco. Rio de Janeiro: autografia, 2017.

MANOEL, E. J.; CARVALHO, Y. M. Pós-graduação na educação física brasileira: a atração (fatal) para a biodinâmica. Educação e Pesquisa, São Paulo, v. 37, n. 2, p. 389-406, 2011.

MASCARENHAS F.; LAZZAROTTI FILHO, A.; VIANNA, L. C. Publicar em inglês ou perecer: a esfinge da internacionalização. Revista Brasileira de Ciências do Esporte, Campinas, v. 40, n. 3, p. 213336, 2018. 
Produtividade na pós-graduação stricto sensu em educação física do Rio de Janeiro... Débora Leonel Peluso - Alan Camargo Silva • Felipe da Silva Triani • Silvio de Cassio Costa Telles • Sílvia Maria Agatti Lüdorf

MATTOS, R. S. A ciência é uma estética da inteligência: o pensamento e a vitalidade das ciências humanas e sociais na educação física. In: TELLES, S.; LÜDORF, S.; GIUSEPPE, E. Pesquisa em educação física: perspectivas sociocultural e pedagógica em foco. Rio de Janeiro: autografia, 2017.

MOLINA NETO, V. et al. Reflexões sobre a produção do conhecimento em educação física e ciências do esporte. Revista Brasileira de Ciências do Esporte, Campinas, v. 28, n. 1, p. 145165, 2006.

PIRES, G. D. L.; POFFO, B. N. A avaliação da pós-graduação em Educação física e suas implicações para os periódicos da área: "publicar ou perecer" vale também para os editores. Motrivivência, Florianópolis, v. 30, n. 54, p. 111-126, 2018. QUADROS, H. M.; AFONSO, M. R.; RIBEIRO, J. A. O cenário da pós-graduação em educação física: contextos e possibilidades na região sul do Brasil. Revista Brasileira de Atividade Física e Saúde, Florianópolis, v. 15, n. 5, p. 76-584, 2013.

RESENDE, H. G.; VOTRE, S. J. O programa de Pós-Graduação Stricto Sensu da Universidade Gama Filho: características, realizações e desafios. Revista Brasileira de Ciências do Esporte, Campinas, v. 24, n. 2, p. 49-73, 2003.

RIGO, L. C.; RIBEIRO, M. R.; HALLAL, P. C. Unidade na diversidade: desafios para a educação física no século XXI. Revista Brasileira de Atividade Física e Saúde, Florianópolis, v. 116, n. 4, p. 339345, 2011.

SACARDO, M. S.; HAYASHI, M. C. P. I. Balanço bibliométrico da produção científica em Educação Física e Educação Especial oriunda de teses e dissertações. Revista Brasileira de PósGraduação, Brasília, v. 9, n. 15, p. 111- 135, 2011.

SAMPAIO, T. M. V. Desafios e perspectivas para a divulgação da pesquisa científica em Educação Física: um processo de construção. Revista Brasileira de Ciências do Esporte, Campinas, v. 36, n. 4, p. 733-739, 2014. 
Produtividade na pós-graduação stricto sensu em educação física do Rio de Janeiro... Débora Leonel Peluso - Alan Camargo Silva • Felipe da Silva Triani • Silvio de Cassio Costa Telles • Sílvia Maria Agatti Lüdorf

SILVA, P. M.; SORIANO, B. J. Qualis periódicos e a produção de capital científico nos programas de pós-graduação em educação física. Movimento, Porto Alegre, v. 20, n. 1, p. 281-304, 2014. TELLES, S.; LÜDORF, S.; GIUSEPPE, E. Pesquisa em educação física: perspectivas sociocultural e pedagógica em foco. Rio de Janeiro: autografia, 2017. Disponível em: https://nespefe.blogspot.com/. Acesso em: 4/10/2018.

TRIANI, F.; TELLES, S. Desafios para a pós-graduação em educação física no Rio de Janeiro. In: Telles, S.; Lüdorf, S.; Giuseppe, E. Pesquisa em educação física: perspectivas sociocultural e pedagógica em foco. Rio de Janeiro, 2017.

VAZ, A, ALMEIDA, F. Q.; BASSANI, J. J. Dificuldades, desafios e dilemas na editoração científica. Revista Brasileira de Ciências do Esporte, Campinas, v. 36, n. 4, p. 752-758, 2014.

VIEIRA, P. V. M.; WAINER, J. Correlações entre a contagem de citações de pesquisadores brasileiros, usando o Web of Science, Scopus e Scholar. Perspectivas em Ciência da Informação, Belo Horizonte, v. 18, n. 3, p. 45-60, 2013.

VILAÇA, M. M.; PALMA, A. Diálogo sobre cientometria, mal-estar na academia e a polêmica do produtivismo. Revista Brasileira de Educação, Rio de Janeiro, v. 18, n. 53, p. 467-484, 2013.

\section{Agradecimento:}

O presente trabalho é derivado da dissertação de mestrado de Débora Leonel Peluso, defendida no Programa de Pós-Graduação em Educação Física da Universidade Federal do Rio de Janeiro e foi realizado com apoio da Coordenação de Aperfeiçoamento de Pessoal de Ensino Superior - Brasil (CAPES) - Código de financiamento 001. 
Produtividade na pós-graduação stricto sensu em educação física do Rio de Janeiro...

Débora Leonel Peluso - Alan Camargo Silva • Felipe da Silva Triani • Silvio de Cassio Costa Telles • Sílvia Maria Agatti Lüdorf

\section{Publisher}

Universidade Federal de Goiás. Faculdade de Educação Física e Dança. Publicação no Portal de Periódicos UFG. As ideias expressadas neste artigo são de responsabilidade de seus autores, não representando, necessariamente, a opinião dos editores ou da universidade. 